\title{
An Effective Method for Load Balancing using Modified Active Monitoring based Ant Clustering
}

\author{
Pragati Rajput \\ Computer Science \\ Adina institute of Science \& Technology \\ Sagar, MP
}

\author{
Ruchika Mishra \\ Assistant Professor \\ Computer Science \\ Adina institute Science \& Technology \\ Sagar, MP
}

\author{
Swati Jain \\ HOD, Assistant Professor \\ Computer Science \\ Adina institute Science \& Technology \\ Sagar, MP
}

\begin{abstract}
Load balancing is a technique of balancing the load on virtual machines for the number of requests coming from the Users to access resources over Data Centers. Here in this paper a new and effectual tactic for Load Balancing over computing is proposed using Modified Active Load Balancing by Ant based Clustering Algorithm. The Existing technique implemented for Load balancing on Cloud Simulators fails to provide efficient load balancing, hence a new approach is proposed where Clustering is done ant based gathering on the basis of Utilization of Virtual Machines. The Proposed methodology provides efficient Throughput and MakeSpan time.
\end{abstract}

\section{Keywords}

Cloud Computing, Ant Colony Optimization. Clustering, Active Monitoring, Load Balancing

\section{INTRODUCTION}

In current years we have understood a growing trend of lasting occurrence of cloud computing in our lives. Nowadays many companies from an extensive variety of branches of industry and science are looking towards a cloud to help simplify their workflows, increase flexibility and adaptability to deliver new and innovative ideas and solutions [1]. Since cloud computing is a new technology .It providing online resources and online storage to the user's .It provide all the data at a lower cost. In cloud computing users can access resources all the time through internet. They need to pay only for those resources as much they use .In Cloud computing cloud provider outsourced all the resources to their client. There are many existing issues in cloud computing. The main tricky is load opposite in cloud calculating. Load harmonizing helps to dispense all loads amongst all the nodes. It also confirms that every calculating reserve is disseminated professionally and honestly. It provides high satisfaction to the users. Load balancing is a comparatively new system that delivers high reserve operation and improved rejoinder time [2,3]. Cloud computing providers qualified rapid growing development in modern years by contribution extremely accessible and scalable services. On the other hand, growing customer resource demand and viable market power the suppliers to make bigger their data centers that show the way to huge power consumption or concern more reasonable resource provision maps that degrades feature of service. For this reason, the cloud providers require the solutions to reduce energy costs and get better resource conditioning.
Within the cloud infrastructure the speed at which this is implemented is considerable. It makes available repairs of infrastructure, platform and software for users and supplies the on-demand services to consumers through Internet [4].

Additionally, a cloud provider has to conceive a big amount of external factors such as geo-distributed infrastructure, weather impact, power outages, etc. Despite improvements in organization sturdiness, many cloud customers motionless face database, hardware, and software downtimes that can lead to loss of submission provision or statistics. Everyone would agree that today's data centers should never go down, and applications should be always available, end-users worldwide need to be able to believe on data center availability for critical data and submission receptiveness at any time [5]. Within the structure of the infrastructure cloud one can reproduce their in-house server circumstances, structure an array of virtual servers and storage explanations to sustain their daily process. The consumer can then right to use their data, applications and resources anywhere in the globe across a series of devices (laptops, tablets, smart phones). Load complementary is a scheme of allocating the entirety weight to the disconnect nodes of the mutual group to the permit networks and assets to make progress the comeback with time of the job with maximum throughput in the scheme [6].

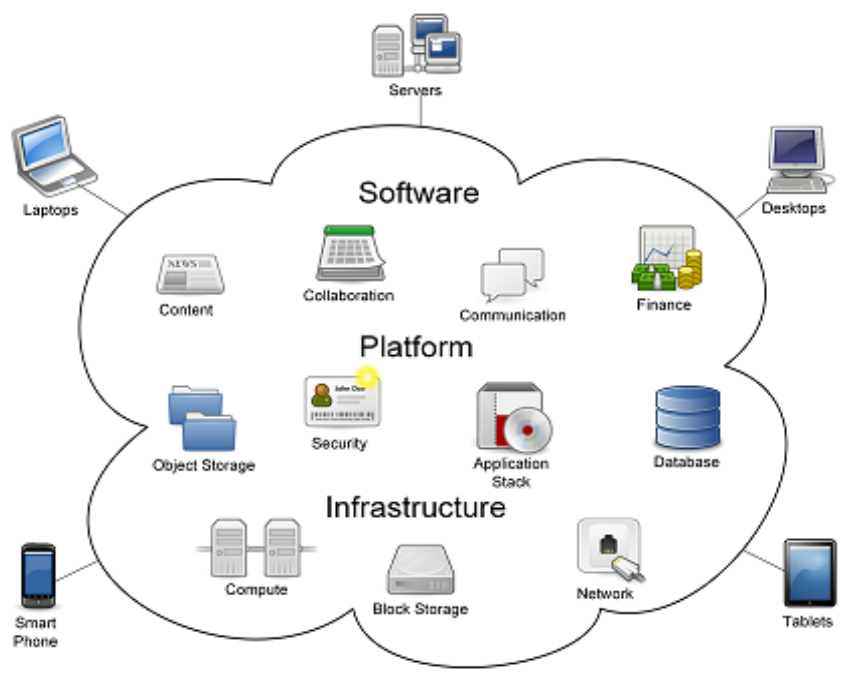

Figure 1: Cloud Computing 
Recent advancements with regard to monitoring techniques within clouds can improve upon this [7], ensuring certain goals are met. The research proposes a organization agenda capable of monitoring the status of workflow executions in the cloud and dynamically allocating resources in response. From a performance unpredictability perspective within clouds this solution can allocate additional resources in response to real time observations in a cloud environment. Virtual machine movement appointment plan is an extensively utilized approach to accomplish load balancing of cloud calculating data middles at present [8], [9]. VMware load balancing explanation is dispersed supply scheduling (DRS) [10]. When DRS select the physical host for the virtual machine it will confirm the load position of each physical host and decide the appointment explanation which can recover the taken as a whole load balance amount. And in the procedure of running a virtual machine, DRS will continuously check the load position of the cluster and utilize VMware VMotion technology to achieve live crusade of virtual apparatuses between different physical servers. Consequently, it can make sure load balancing and wellorganized use of physical resource of the complete cluster. In [11], Piao and Yan have suggested a system aware simulated machine assignment and migration move toward to reducing the data transfer instance use and improving the general application presentation of the cloud data center. On the other hand, this move toward almost certainly effects in a comparatively lower use of resource of physical hosts and increases the process expenditure of the cloud data center.

\section{LITERATURE SURVEY}

In this paper [12], here author proposed LB-BC method that is based on heuristic job deployment method, which is utilized to organize job requests accepted by the cloud data center into optimal objective physical hosts in the IaaS cloud computing data center. Its algorithm is what joins Bayes theorem with clustering. It has accomplished the general load balancing of the complete arrangement from the viewpoint of cloud data centers' long time operations and consequently to get better the presentation and effectiveness. The job deployment procedure of LB-BC is revealed in Fig. 3. Initially, these physical hosts each of which has a superior outstanding resource quantity than the maximum demanded resource quantity of all job requests can be investigated out to represent a novel applicant set to assemble the presentation restriction while manufacture LB-BC have the possible of accomplishing the durable load balancing. Then subsequent, the $\mathrm{k}$ corporeal crowds in the set of physical hosts can be observed as k objects staying for being clustered. Each physical host in the particular set is given to a prior possibility.

The subsequent probability of each physical host's management jobs can be computed all the way through Bayes theorem. This possibility can be observed as an characteristic of each object while the continuing CPU resource quantity and the outstanding memory reserve quantity of each physical host can be observed as the other two attributes. The comparison degree assessments between physical hosts are computed according to the three characteristics of each physical host. A threshold assessment is established on the origin of these comparison degree values.

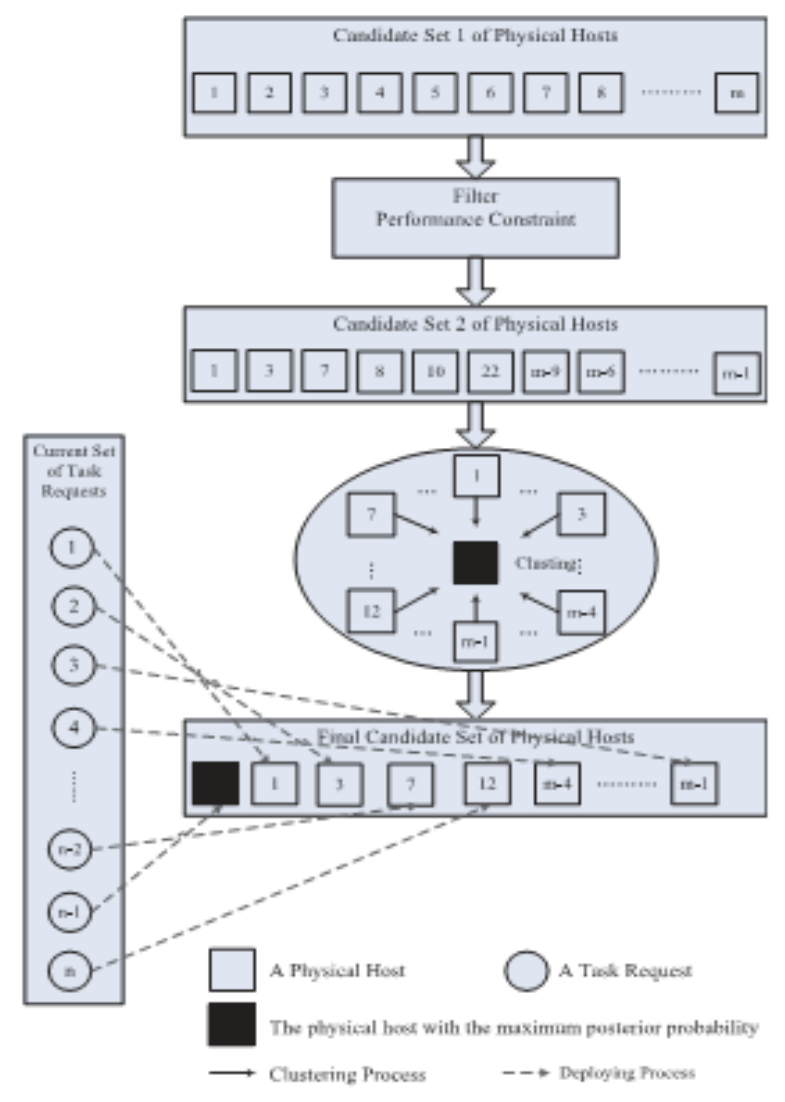

Figure-2 the process of LB-BC task deployment

The physical hosts whose comparison degree values between them are surrounded by the given threshold can be observe as the optimal clustering to form the absolute set of applicant physical hosts. to end with, the jobs are positioned on the hosts in the finishing set. And the clustering process of corporeal congregations in the cloud information center is the progression of discovering the optimal physical hosts for executing jobs.

Sushil Kumar et.al [13] proposed a soft subtracting method which uses the system of natural selection plan A unassuming Genetic Algorithm is collected of three processes: genetic operation, selection, and replacement operation. The benefit of this method is that it can feel a huge search space appropriate to composite objective function and can keep away from being entrapped in locally optimal solution. A generation is an assortment of artificial creatures i.e. strings. In every novel generation, a set of strings is generated using information from the earlier ones. Infrequently, a novel dimension is attempt for good calculate. According to Genetic Algorithms are randomized, but they are not easy random walks. They proficient exploit earliest substance to vulnerability on novel search views with conventional expansion. The efficiency of the GA depends in suitable mix of examination and use.

R. Stanojevic et.al [14] planned a mechanism CARTON for haze control. It combines the exploit of load balancing (LB) and distributed rate limiting (DRL). LB is utilized for uniformly distributing the jobs to different wait persons so that the related costs can be diminished and DRL is used to make unquestionable that the profits are dispersed in a technique to maintain a reasonable hold back distribution. With very low computation and announcement upstairs, this algorithm is easy and straightforward to put into operation. 
Zhao et.al [15] proposed his methods with a position of the challenging of intra-cloud load balancing between physical people attending worships by adaptive live immigration of virtual equipments. A load balancing representation is measured and useful in teaching to decrease virtual machines relocation time by communal contents to balance load between servers leavening to their workstation or IO usage and to maintain simulated machineries zero-downtime in the process.

Finally, there is modern effort for resource location in clouds [16], which can be understand as a publish/subscribe scheme, although with moderately understandable differences. Here nodes query for a reserve with confident attributes and are forwarded to a measurement of the cloud that holds the resources with demanded properties. This effort also uses a peer example repair to build an arrangement and an unstructured superimpose. In the unstructured superimpose, reserves with comparable attributes are positioned secure to one an additional. On the other hand, does not security and actually does not require that all the nodes with the uncertainty properties are establish. However, in Vitis, they create confident that all the subscribers are originate and notified of the issued occasion. Additionally, is not appropriate for event distribution for it implements an important load on the nodes in the structured superimpose.

\section{PROPOSED METHODOLOGY}

The proposed methodology implemented here consists of Improving Ant based Clustering Scheduling which is based on the concept of Clustering the Pheromone values at each node of the cloud.

1. First of all create Cloud Simulation environment.

2. set the number of user 'Ui' and a Number of resources 'Ri' to access.

The model is fully distributed, i.e. every node behaves separately as well as each ant or agent, and this denotes that every node or ant is autonomous. Figure represents the table attached to each node or ant. In the classical, each protuberance comprises a table that contains evidence about other nodes in the system. At the original national, the table entrances are Null. In each ant excursion, the ant will transport the rationalized evidence about all nodes that the ant has been passed throughout. Upon entrance of the ant at every protuberance, the subsequent actions will be done:

Undertake a system is arrangement and a quantity of packages send from foundation to endpoint and the worth of pheromone dropped at each nodes and shortest path is selected using MaxMin, Rank based and Fuzzy System.

1. Suppose ' $N$ ' of packets to be send from Source ' $S$ ' to destination ' $D$ '.

2. Initialize all the pheromone table of the node to zero.

3. When first packet is send from one node to another pheromone value is updated accordingly at that node and update all the tables of the network.

4. Proposed methodology uses the limitation $\mathrm{s}$ of the existing ant based techniques; hence at each node of the network the possibility of various paths from that node to next node is computed and updated.

5. After first iteration the value of the value of pheromone is calculated at each node of the network.
6. More value of pheromone attracts more ants, hence the next packet is send to that particular nodes where pheromone value is maximum.

\begin{tabular}{|c|c|}
\hline Algori & $\mathrm{hm}$ \\
\hline 1. & Initialize all the parameters. \\
\hline 2. & $\begin{array}{l}\text { If ' } N \text { ' quantity of packages send from foundation to } \\
\text { destination. }\end{array}$ \\
\hline 3. & For every Packet 'P' send from Node 'N1' $\rightarrow$ 'N2'. \\
\hline 4. & Count initial Tsp \\
\hline 5. & $\begin{array}{l}\text { Deposit the assessment of pheromone charge at Node } \\
\text { 'N1' \& 'N2'. }\end{array}$ \\
\hline 6. & $\mathrm{Tp}=\mathrm{Tp}+\mathrm{P}$ \\
\hline 7. & If there are 'Npath' from one node 'N1' $\rightarrow$ 'N2' \\
\hline 8. & Check the pheromone table of $\mathrm{Tm}$ and $\mathrm{Tr}$ and $\mathrm{Tf}$. \\
\hline & $\begin{array}{l}\text { If for every algorithm through pathway from one } \\
\text { protuberance to next node is same }\end{array}$ \\
\hline 10 & Choose that node as the next node. \\
\hline & Sht $=$ Initail Node + Sht \\
\hline & $\mathrm{Tp}=\mathrm{Tp}+\mathrm{p}$ \\
\hline & Else \\
\hline & $\begin{array}{l}\text { Check the two best algorithms having unswerving } \\
\text { track from one protuberance to next as same. }\end{array}$ \\
\hline & Choose that node as the next node. \\
\hline & Sht $=$ Previous node + Sht \\
\hline & $\mathrm{Tp}=\mathrm{T} p+\mathrm{p}$ \\
\hline & If a stopping criteria is met, then \\
\hline & $\begin{array}{l}\text { Cluster Resources conferring to their Pheromone } \\
\text { Morals in the Pheromone Table on VMs }\end{array}$ \\
\hline & Else \\
\hline & Empty Pheromone Table, go to 2 \\
\hline & End if \\
\hline & End \\
\hline & Count Time after sending 'N' packets Tsp1; \\
\hline & TotalTime $=$ Tsp1-Tsp; \\
\hline
\end{tabular}

\section{PROPOSED METHODOLOGY}

Ant based Gathering is first planned by Deneubourget. Al. and rendering to him the ants can pace haphazardly on the workspace by picking and dropping of data from one place to another, but the as soon as they move from one place to another a chemical named Pheromone is deposited which attracts other ants to move at the same place. Here we planned an wellorganized system of applying ant based clustering for the Optimization of VM Scheduling in the Cloud Computing Environment.

$\mathrm{T}=\{\mathrm{t} 1, \mathrm{t} 2 \ldots . \mathrm{tn}\}$ represents the tasks waiting to be scheduled per unit of time, $n$ is the number of tasks.

$\mathrm{N}=\{\mathrm{n} 1, \mathrm{n} 2, \mathrm{n} 3 \ldots \mathrm{nm}\}$ represents the set of nodes in the cloud system, assuming that the cloud system has m nodes. For the cloud computing system, ni represents the computing resources 
on the ni; For the Cloud Storage System, ni represents the data on a ni.

$\mathrm{V}=[\mathrm{v} 1, \mathrm{v} 2 \ldots \mathrm{vn}]$ represents the task scheduling vectors or a scheduling scheme. For Cloud storage system, i v represents the i-th task of data is provided by resources nodes that represented by i v value, and the length of the vector is the total quantity of scheduling tasks per unit time.

For example, a task scheduling vector [5, 1, 3, 2, 1, 6], the length of this vector is 6 , and represents needs to schedule task number is 6 per unit of time. The value based on the position of No. 1 is 5 , represent the data of the task 1 is provided by the system node 5. So, the data of task 2 and 5 are provided by the node 1 ; the data of task 3 is provided by the node 3 ; the data of task 4 is provided by the node 2; the data of task 6 is provided by the node 6 . For cloud computing system, it is on behalf of a task placed in a node.

The Probability of Pick it up can be assumed by the subsequent purpose:

$$
P_{\text {pick }}=\left(\frac{K^{+}}{K^{+}+f}\right)^{2}
$$

Where, ' $\mathrm{f}$ ' is an estimation of the fraction of nearby hosts occupied by objects of the same type, and $\mathrm{K}^{+}$is a constant. The Probability thus decreases with ' $\mathrm{f}$ ', from 1 (when $\mathrm{f}=0$ ) to $1 / 4$ (when $\mathrm{f}=\mathrm{K}^{+}$),

The Probability of Drop can be assumed by the subsequent meaning;

$$
P_{\text {drop }}=\left(\frac{f}{K^{-}+f}\right)^{2}
$$

Where, ' $\mathrm{f}$ ' is same and $\mathrm{K}^{-}$is also constant.

\section{INITIAL PHEROMONE LAYING}

This is initialization step. Every location $(i, j)$ with an object on the grid will be assigned a pheromone $\tau$ ij based on the surrounding. Let $\Delta \tau$ be the amount of pheromone change. The presence of similar objects in the surroundings increases the pheromone trail on the location by $\Delta \tau$ and a dissimilar object decreases the trail by $\Delta \tau$.

\section{CLUSTER CONSTRUCTION}

Ants move randomly on the grid. If an unloaded ant meets an object and finds pheromone on that location below the threshold value, it picks it up. If encumbered ant originates to a position with pheromone value greater than the threshold and its load matches with the object on that location, it drops in neighborhood of location with probability.

\section{PHEROMONE UPDATION}

On a pick/drop action, the pheromone on that location and the surrounding location will be updated. On Pickup, $\tau_{\mathrm{ij}}=0$ and pheromone in the surrounding cells containing the similar object will be decreased and containing dissimilar objects will be increased. On Drop, and pheromone in the surrounding cells containing the similar object will be increased and containing dissimilar objects will be decreased.

1. Initialize the Counter $\mathrm{C}=0$

2. Repeat until all the ants move

3. Set $\mathrm{s}=\mathrm{s}+1$, where $\mathrm{s}=1$ as index

4. For $\mathrm{k}=1$ to $\mathrm{m}$ do

5. Choose the VM to move with the probability $P_{i j}^{k}(t)$
6. Move the kth ant over the $\mathrm{VM} \mathrm{j}$

7. Insert the $\mathrm{VM} \mathrm{j}$ into the Pheromone Table

8. End for

9. End repeat

10. For every edge $(I, j)$ do

11. $\Delta \tau_{i j}=\sum_{k=1}^{m} \Delta \tau_{i j}^{k}$

12. Compute $\tau_{i j}(t+1)=(1-\rho) \tau_{i j}(t)+\Delta \tau_{i j}$

13. Set $\Delta \tau_{i j}=0$

14. End for

15. Set $\mathrm{t}=\mathrm{t}+1$

16. If a stopping criteria is met, then

17. Cluster Resources conferring to their Pheromone Principles in the Pheromone Table on VMs

18. Else

19. Empty Pheromone Table, go to 2

20. End if

\section{RESULT ANALYSIS}

The table shown below is the analysis and comparison of MakeSpan time between the prevailing Load opposite system and the proposed methodology. The Analysis is done on the basis of number of requested tasks and hence MakeSpan time is computed for the existing and the proposed methodology.

Table 1: Analysis of MakeSpan Time

MakeSpan (S)

\begin{tabular}{|c|c|c|}
\hline No. of Requested Tasks & Existing Work & Proposed Work \\
\hline 5 & 300 & 273 \\
\hline 10 & 350 & 325 \\
\hline 15 & 400 & 374 \\
\hline 20 & 500 & 462 \\
\hline 25 & 750 & 728 \\
\hline 30 & 1000 & 838 \\
\hline
\end{tabular}

The table shown below is the analysis and comparison of Energy Consumption between the present Load matching system and the proposed methodology. The Analysis is done on the basis of number of Hosts and hence Energy Consumption is computed for the existing and the proposed methodology.

Table 2. Analysis of Energy Consumption

\begin{tabular}{|c|c|c|c|}
\hline & \multicolumn{3}{|c|}{ Energy Consumption (Kwh) } \\
\hline $\begin{array}{c}\text { No. of } \\
\text { Hosts }\end{array}$ & No. of Vm's & $\begin{array}{c}\text { Existing } \\
\text { Work }\end{array}$ & $\begin{array}{c}\text { Proposed } \\
\text { Work }\end{array}$ \\
\hline 10 & 5 & 1.53 & 1.16 \\
\hline
\end{tabular}




\begin{tabular}{|c|c|c|c|}
10 & 10 & 1.55 & 1.27 \\
\hline 10 & 15 & 1.87 & 1.72 \\
\hline 10 & 20 & 2.27 & 2.19 \\
\hline 10 & 25 & 2.46 & 2.32 \\
\hline 10 & 30 & 2.65 & 2.56 \\
\hline
\end{tabular}

\begin{tabular}{|l|l|l|}
700 & 0.06 & 0.03 \\
\hline 800 & 0.07 & 0.04 \\
\hline 900 & 0.06 & 0.03 \\
\hline 1000 & 0.05 & 0.02 \\
\hline
\end{tabular}

\section{CONCLUSION}

Cloud computing has emerged as a cost-effective means of delivering indicator to calculate resources. It supports a pay-asyou-go model of computation in which application owners (cloud consumers) pay only for the resources used. The Proposed Methodology here for Load Balancing using Ant based Clustering provides efficient Throughput and Makespan time. The Existing Technique implemented for Load Balancing using Nave Bayes Clustering provides efficient Load balancing over clouds, but Ant based Clustering approach for Active Monitoring Approach gives more improved Throughput and Efficient MakeSpan time.

Table 3: Analysis of Throughput

\begin{tabular}{|c|c|c|}
\hline & \multicolumn{2}{|c|}{ Throughput } \\
\hline Time (S) & Existing Work & $\begin{array}{c}\text { Proposed } \\
\text { Work }\end{array}$ \\
\hline 200 & 1.54 & 1.84 \\
\hline 400 & 2.36 & 2.74 \\
\hline 600 & 2.48 & 2.89 \\
\hline 800 & 2.63 & 3.12 \\
\hline 1000 & 2.75 & 3.23 \\
\hline 1200 & 2.81 & 3.34 \\
\hline 1400 & 3.2 & 3.56 \\
\hline
\end{tabular}

The table shown below is the analysis and comparison of Standard Deviation between the prevailing Load balancing method and the proposed methodology. The Analysis is done on the basis of number of Time and hence Standard Deviation is computed for the existing and the proposed methodology.

Table 4: Analysis of Standard Deviation

\begin{tabular}{|c|c|c|}
\hline & \multicolumn{2}{|c|}{ Standard Deviation } \\
\hline Time (S) & Existing Work & Proposed Work \\
\hline 100 & 0.24 & 0.22 \\
\hline 200 & 0.27 & 0.23 \\
\hline 300 & 0.25 & 0.2 \\
\hline 400 & 0.19 & 0.15 \\
\hline 500 & 0.16 & 0.11 \\
\hline 600 & 0.17 & 0.13 \\
\hline
\end{tabular}

\section{REFERENCES}

[1] F. Leymann C. Fehling and R. Retter et al. Cloud Computing Patterns: Fundamentals to Design, Build, and Manage Cloud Applications. Springer, 2014.

[2] Mell, Peter and Grance, Tim, "The NIST definition of cloud computing", National Institute of Standards and Technology, 2009, vol53, pages50, Mell2009.

[3] S. S. Moharana, R. D. Ramesh, D. Powar, "Analysis of load balancers in cloud computing" International Journal of Computer Science and Engineering,2013,vol 2,pages 101-108.

[4] Rahman, Mazedur, Samira Iqbal, and Jerry Gao. "Load Balancer as a Service in Cloud Computing." In Service Oriented System Engineering (SOSE), 2014 IEEE 8th International Symposium on, pp. 204-211. IEEE, 2014.

[5] Evolven's IT Operations Analytics. Downtime, outages and failures - understanding their true costs, September 2013. Accessed: 2014-06-05.

[6] Rajkumar Buyya. Market-oriented cloud computing: Vision, hype, and reality of delivering computing as the $5^{\text {th }}$ utility. In CCGRID, page 1, 2009.

[7] Vincent C Emeakaroha, Paweł P Łabaj, Michael Maurer, Ivona Brandic, and David $\mathrm{P}$ Kreil. Optimizing bioinformatics workflows for data analysis using cloud management techniques. In Proceedings of the $6^{\text {th }}$ workshop on Workflows in support of large-scale science, pages 37-46. ACM, 2011.

[8] M. Armbrust, A. Fox, R. Griffith, A. D. Joseph, R. Katz, A. Konwinski, G. Lee, D. Patterson, A. Rabkin, I. Stoica, and M. Zaharia, "A view of cloud computing," Commun. ACM, vol. 53, no. 4, pp. 50-58, 2010.

[9] R. Moreno-Vozmediano, R. S. Montero, and I. M. Llorente, "Key challenges in cloud computing: enabling the future internet of services," IEEE Int. Comput., vol. 17, no. 4, pp. 18-25, Jul./Aug. 2013.

[10] A. Gulati, A. Holler, M. Ji, G. Shanmuganathan, C. Waldspurger, and X. Zhu, "VMware distributed resource management: Design, implementation and lessons learned," VMware Tech. J., vol. 1, no. 1, pp. 45-64,Mar. 2012. 
[11] J. T. Piao and J. Yan, "A network-aware virtual machine placement and migration approach in cloud computing," in Proc. $9^{\text {th }}$ IEEE Int. Conf. Grid Cooperative Comput., 2010, pp. 87-92

[12] Jia Zhao, Kun Yang,” A Heuristic Clustering-Based Task Deployment Approach for Load Balancing Using Bayes Theorem in Cloud Environment", IEEE Transaction on Parallel and Distributed Systems, Volume 27, Issue 2, February 2016.

[13] Sushil Kumar,Deepak Singh Rana "Dynamic Load Balancing Algorithms in Cloud Environment: A Survey"
International Journal of Computer Applications (0975 8887) Volume 129 - No.6, November2015.

[14] Stanojevic R. and Shorten R.(2009) IEEE ICC, 1-6.

[15] Zhao Y. and Huang W. (2009) 5th International Joint Conference on INC, IMS and IDC, 170-175.

[16] J. Alveirinho, J. Paiva, J. Leitao, and L. Rodrigues, "Flexible and Efficient Resource Location in Large-Scale Systems," The 4th ACM SIGOPS/SIGACT Workshop on Large Scale Distributed Systems and Middleware, 2010. 